\title{
Cadmium(II) and Zinc(II) Complexes of $S$-Confused Thiaporphyrin
}

Michał J. Chmielewski, Miłosz Pawlicki, Natasza Sprutta, Ludmiła Szterenberg and Lechosław Latos-Grażyński*

Department of Chemistry, University of Wrocław, 50383 Wrocław, Poland

\section{Supporting Informations}

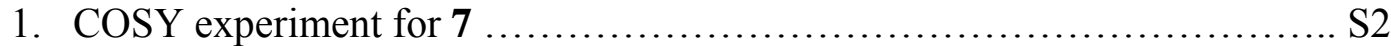

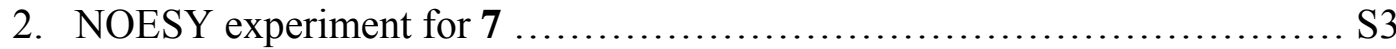

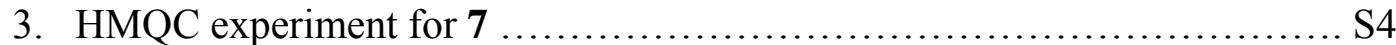

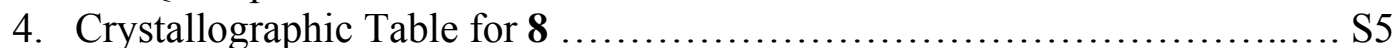

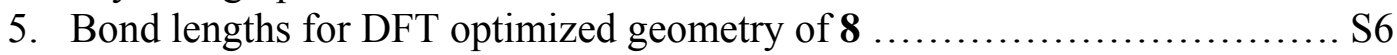

6. Cartesian coordinates (xyz) for DFT optimized geometry of $8 \ldots \ldots \ldots \ldots$. S7

7. Coordinates of Bond Critical Points (BCPs; AIM analysis) for 8 ............ S9

8. Coordinates of Ring points (AIM analysis) for 8 ........................... S11

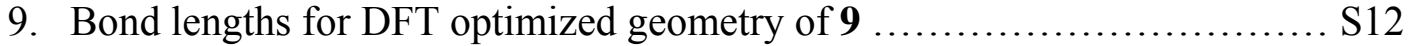

10. Cartesian coordinates (xyz) for DFT optimized geometry of $9 \ldots \ldots \ldots \ldots . . . . .513$

11. Parameters of selected Bond Critical Points for 9 (BCPs; AIM analysis ) ... S15 

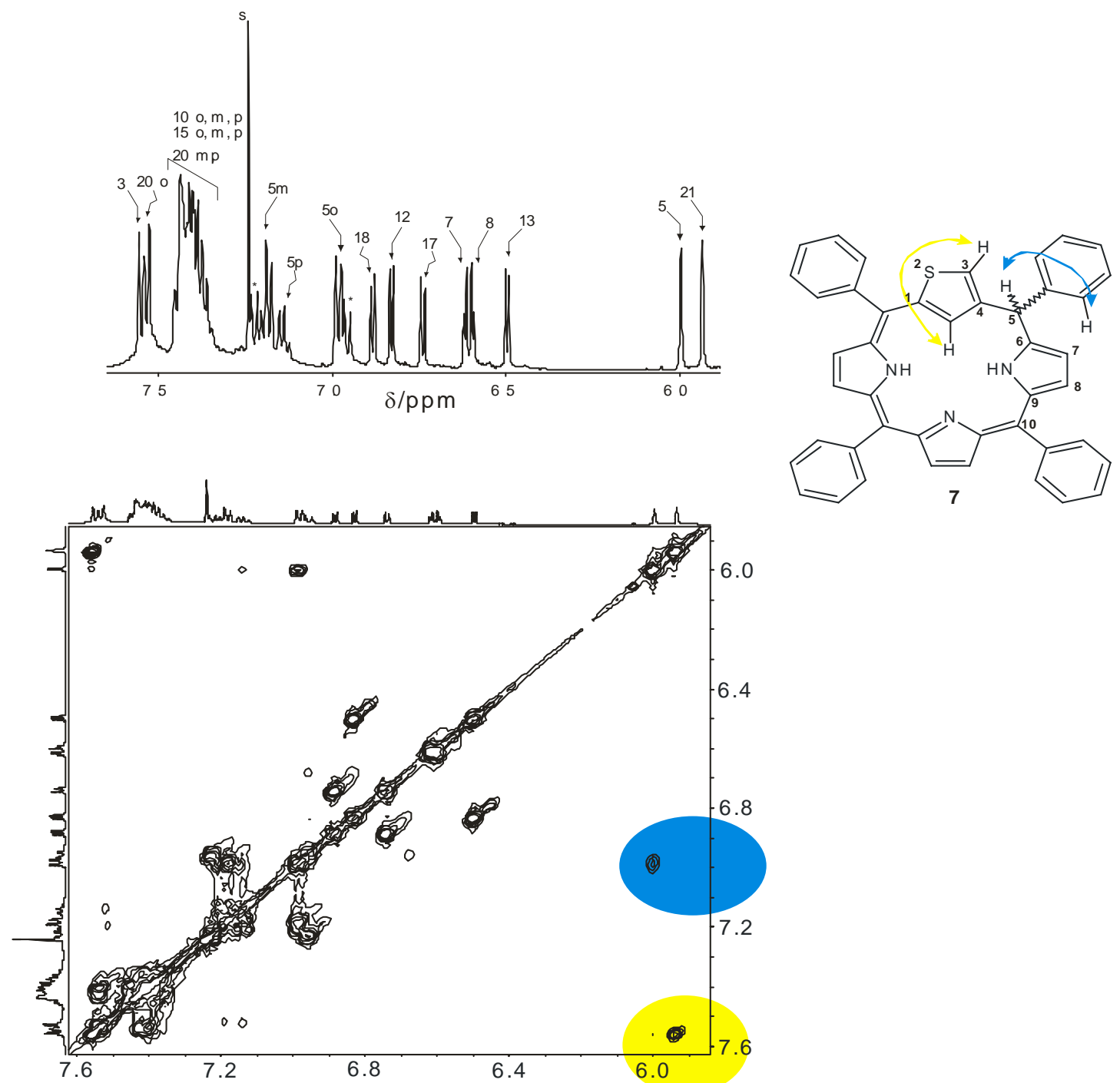

Figure S1. COSY experiment for 7 

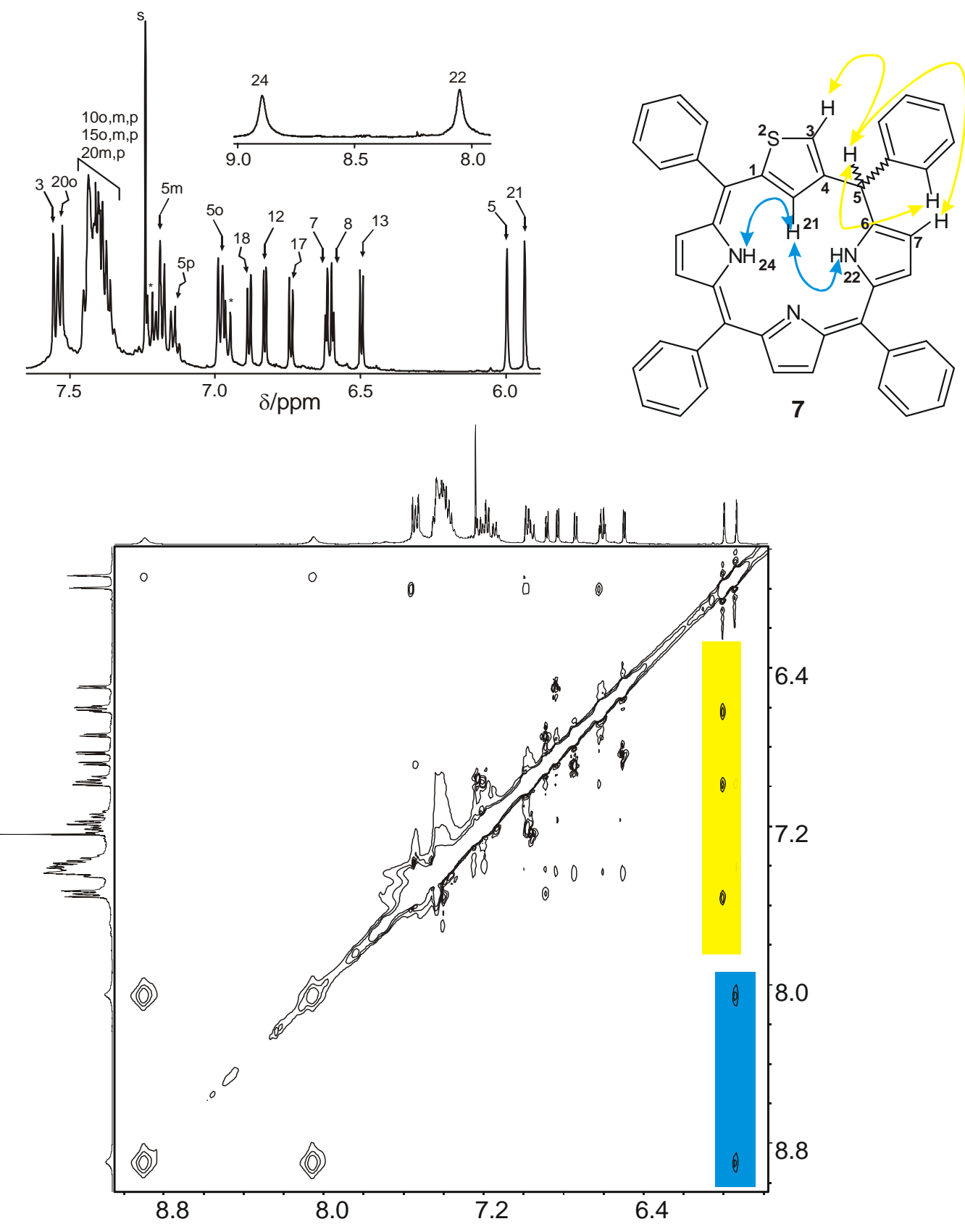

Figure S2. NOESY experiment for 7. 

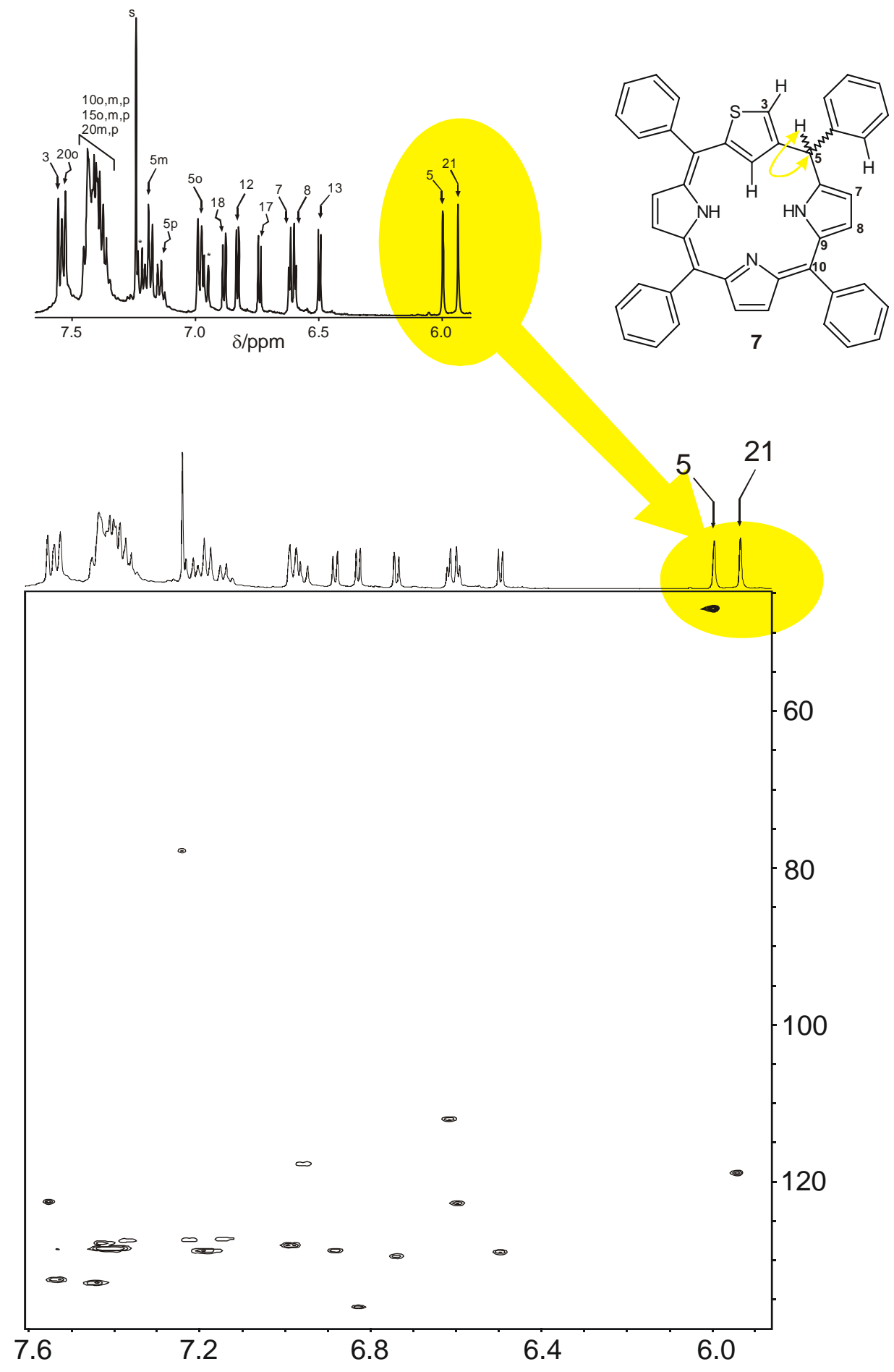

Figure S3. HMQC experiment for 7. 
Table S1.

\begin{tabular}{|c|c|}
\hline Compound & $\mathbf{8} \cdot \mathrm{CH}_{2} \mathrm{Cl}_{2} \cdot 0.5 \mathrm{C}_{5} \mathrm{H}_{12}$ \\
\hline crystals grown by & slow diffusion of pentane into $\mathrm{CH}_{2} \mathrm{Cl}_{2}$ solution \\
\hline crystal habit & irregular green block \\
\hline formula & $\mathrm{C}_{47.5} \mathrm{H}_{36} \mathrm{~N}_{3} \mathrm{~S} \mathrm{Cd} \mathrm{Cl}_{3}$ \\
\hline fw & 899.6 \\
\hline$a, \AA$ & $9.6718(19)$ \\
\hline$b, \AA$ & $14.229(3)$ \\
\hline$c, \AA$ & $15.428(3)$ \\
\hline$\alpha,^{\circ}$ & $76.06(3)$ \\
\hline$\beta,^{\circ}$ & $86.31(3)$ \\
\hline$\gamma,^{\circ}$ & $76.17(3)$ \\
\hline$V, \AA^{3}$ & $2001.0(7)$ \\
\hline$Z$, & 2 \\
\hline$D_{\text {calc }} \mathrm{g} \cdot \mathrm{cm}$ & 1.493 \\
\hline crystal system & triclinic \\
\hline space group & $P-1$ \\
\hline$\mu, \mathrm{mm}$ & 0.837 \\
\hline absorption correction & not applied \\
\hline $\mathrm{T}, \mathrm{K}$ & $100(2)$ \\
\hline$\theta$ range & $2.92 \leq \theta \leq 28.38$ \\
\hline$h k l$ range & $\begin{array}{l}-12 \leq h \leq 11 \\
-18 \leq k \leq 18 \\
-19 \leq l \leq 20\end{array}$ \\
\hline \multicolumn{2}{|l|}{ reflections } \\
\hline measured & 17003 \\
\hline unique, $\mathrm{I}>2 \sigma(\mathrm{I})$ & 9107 \\
\hline parameters/restraints & $510 / 0$ \\
\hline $\mathrm{R} 1$ & 0.0764 \\
\hline wR2 & 0.1476 \\
\hline $\mathrm{S}$ & 0.942 \\
\hline
\end{tabular}




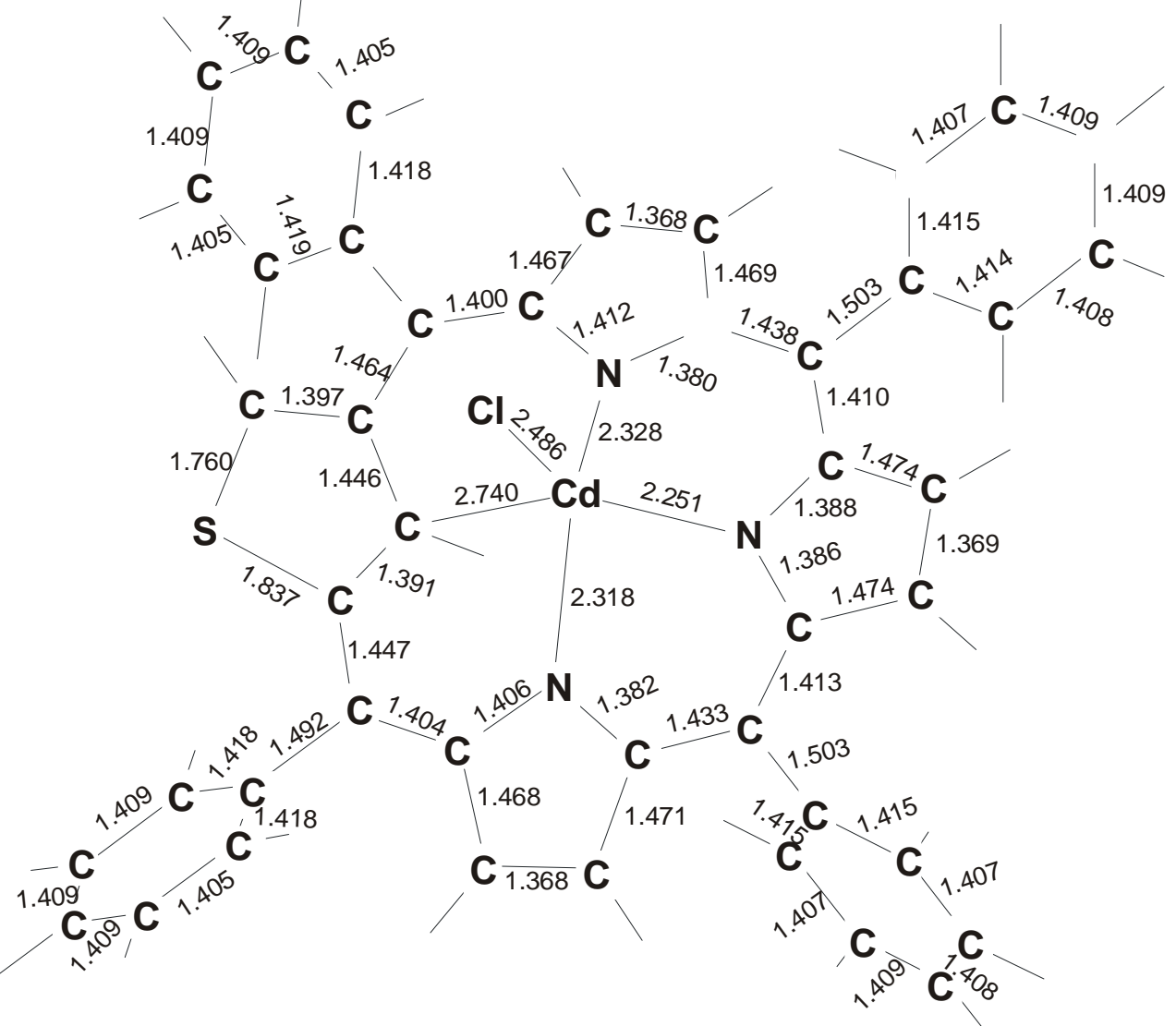

Figure S4. Bond lengths for DFT optimized geometry of 8 
Table S2. Cartesian coordinates for DFT optimized geometry of 8.

\begin{tabular}{|c|c|c|c|}
\hline S & -4.333948 & 0.027955 & 0.755829 \\
\hline $\mathrm{C}$ & -3.712042 & 1.663223 & 0.564490 \\
\hline $\mathrm{C}$ & -2.531850 & 1.732411 & -0.180469 \\
\hline $\mathrm{C}$ & -1.823716 & 3.000148 & -0.368988 \\
\hline $\mathrm{C}$ & -0.429852 & 3.089986 & -0.459294 \\
\hline $\mathrm{C}$ & 0.315292 & 4.295859 & -0.836340 \\
\hline $\mathrm{C}$ & 1.646776 & 3.988480 & -0.767726 \\
\hline $\mathrm{C}$ & 1.755102 & 2.581730 & -0.357096 \\
\hline $\mathrm{C}$ & 3.021954 & 1.917007 & -0.209977 \\
\hline $\mathrm{C}$ & 3.207944 & 0.524652 & -0.091916 \\
\hline $\mathrm{C}$ & 4.504432 & -0.170339 & -0.004584 \\
\hline $\mathrm{C}$ & 4.245438 & -1.514848 & -0.001916 \\
\hline $\mathrm{C}$ & 2.783209 & -1.679255 & -0.085208 \\
\hline $\mathrm{C}$ & 2.093643 & -2.908112 & -0.195584 \\
\hline $\mathrm{C}$ & 0.674472 & -3.050120 & -0.338362 \\
\hline $\mathrm{C}$ & 0.036298 & -4.311964 & -0.743232 \\
\hline $\mathrm{C}$ & -1.311602 & -4.087240 & -0.813697 \\
\hline $\mathrm{C}$ & -1.536903 & -2.683330 & -0.449942 \\
\hline $\mathrm{C}$ & -2.792951 & -2.058938 & -0.389715 \\
\hline $\mathrm{C}$ & -2.931331 & -0.627018 & -0.233703 \\
\hline $\mathrm{C}$ & -2.120954 & 0.426127 & -0.644669 \\
\hline $\mathrm{N}$ & 0.491049 & 2.051990 & -0.198547 \\
\hline $\mathrm{N}$ & 2.194917 & -0.424521 & -0.092027 \\
\hline $\mathrm{N}$ & -0.295702 & -2.078959 & -0.181801 \\
\hline $\mathrm{Cd}$ & 0.164847 & -0.027767 & 0.795327 \\
\hline $\mathrm{Cl}$ & -0.409688 & 0.125375 & 3.209542 \\
\hline $\mathrm{C}$ & 4.247506 & 2.787230 & -0.237627 \\
\hline $\mathrm{C}$ & 4.470901 & 3.738168 & 0.785844 \\
\hline $\mathrm{C}$ & 5.616176 & 4.554916 & 0.773000 \\
\hline $\mathrm{C}$ & 5.197148 & 2.683577 & -1.280702 \\
\hline $\mathrm{C}$ & 6.339640 & 3.505059 & -1.299443 \\
\hline $\mathrm{C}$ & 6.555326 & 4.442659 & -0.271012 \\
\hline $\mathrm{C}$ & -2.651347 & 4.239424 & -0.403911 \\
\hline $\mathrm{C}$ & -2.321425 & 5.367710 & 0.389596 \\
\hline $\mathrm{C}$ & -3.118807 & 6.524407 & 0.363466 \\
\hline $\mathrm{C}$ & -4.261027 & 6.581802 & -0.458942 \\
\hline $\mathrm{C}$ & -4.606446 & 5.465206 & -1.246054 \\
\hline $\mathrm{C}$ & -3.816784 & 4.303892 & -1.211007 \\
\hline $\mathrm{C}$ & -4.036746 & -2.881464 & -0.435551 \\
\hline $\mathrm{C}$ & -4.188350 & -4.028378 & 0.383693 \\
\hline $\mathrm{C}$ & -5.370872 & -4.786246 & 0.346864 \\
\hline $\mathrm{C}$ & -6.425102 & -4.416050 & -0.511054 \\
\hline $\mathrm{C}$ & -6.290392 & -3.274564 & -1.325663 \\
\hline $\mathrm{C}$ & -5.111985 & -2.510832 & -1.282413 \\
\hline $\mathrm{C}$ & 2.908940 & -4.170195 & -0.224905 \\
\hline $\mathrm{C}$ & 2.761349 & -5.140013 & 0.794615 \\
\hline
\end{tabular}

Chmielewski, Pawlicki, Sprutta, Szterenberg, Latos-Grażyński 


$\begin{array}{lrrr}\mathrm{C} & 3.521648 & -6.323583 & 0.779047 \\ \mathrm{C} & 4.438424 & -6.563824 & -0.263109 \\ \mathrm{C} & 4.588660 & -5.609188 & -1.287670 \\ \mathrm{C} & 3.832593 & -4.422626 & -1.266257 \\ \mathrm{H} & -0.129086 & 5.226747 & -1.155799 \\ \mathrm{H} & 2.482617 & 4.629089 & -1.005905 \\ \mathrm{H} & 5.467933 & 0.313323 & 0.056042 \\ \mathrm{H} & 4.960397 & -2.321478 & 0.062391 \\ \mathrm{H} & 0.563543 & -5.226184 & -0.971608 \\ \mathrm{H} & -2.079774 & -4.777994 & -1.127875 \\ \mathrm{H} & -1.311507 & 0.285247 & -1.352824 \\ \mathrm{H} & 3.753476 & 3.822808 & 1.598555 \\ \mathrm{H} & 5.775595 & 5.271582 & 1.575199 \\ \mathrm{H} & 5.029189 & 1.971352 & -2.084973 \\ \mathrm{H} & 7.054333 & 3.415513 & -2.114329 \\ \mathrm{H} & -1.461354 & 5.318141 & 1.051326 \\ \mathrm{H} & -2.856156 & 7.372378 & 0.991135 \\ \mathrm{H} & -4.876372 & 7.477959 & -0.479856 \\ \mathrm{H} & -5.485810 & 5.500341 & -1.884590 \\ \mathrm{H} & -4.085781 & 3.450196 & -1.827571 \\ \mathrm{H} & -3.391947 & -4.299702 & 1.071198 \\ \mathrm{H} & -5.474010 & -5.654031 & 0.993591 \\ \mathrm{H} & -7.339911 & -5.003095 & -0.538828 \\ \mathrm{H} & -7.098827 & -2.982098 & -1.991323 \\ \mathrm{H} & -5.011238 & -1.636527 & -1.920400 \\ \mathrm{H} & 2.062594 & -4.954939 & 1.606916 \\ \mathrm{H} & 3.401181 & -7.051505 & 1.577924 \\ \mathrm{H} & 5.287619 & -5.788134 & -2.101457 \\ \mathrm{H} & 3.943278 & -3.695657 & -2.067250 \\ \mathrm{H} & -4.215455 & 2.482020 & 1.058358 \\ \mathrm{H} & 7.439624 & 5.075440 & -0.283056 \\ \mathrm{H} & 5.024875 & -7.479478 & -0.276827\end{array}$


Table S3. Bond Critical Points (BCP) for 8.

$-7.60348604020365$

$-5.858556962455$

$-4.10484150552821$

$-2.12938798238787$

$-0.109637285869476$

$-0.0793033311908038$

1.86766743834497

3.23763868753853

$-1.89337929342926$

2.34771707504291

4.53097763010092

0.600382346077354

5.8709196482148

7.32917548109028

$-0.145465499747945$

2.24743237836475

5.28553559812437

8.28605304346515

6.67688206709521

4.81438671167874

4.58273149454569

$-0.220088774825927$

2.64111745795786

0.676589194618492

$-1.20403365139302$

0.523407949839088

$-2.71325301954233$

$-6.82498300508657$

$-4.73190456152243$

$-4.37773988387593$

$-1.92506488073983$

$-4.09010890432096$

$-5.41272088103775$

$-4.23546685562215$

6.88046049552901

8.23522640387383

9.53620272775717

8.93462187649384

10.9054519808477

11.5047236722592

12.1895851519371

$-4.68133166313394$

$-5.1431677840289$

0.0611646917915294

$-6.96830575171751$

$-6.12524601388911$

$-8.37707155983147$
1.60685781707355

3.21610164409855

4.48252817269727

5.7410040910069

7.0211037815469

5.02618153678959

7.84508644478165

6.24290125214655

0.366642541180718

4.47971965071424

4.22039138430264

1.92492993096251

2.32753749766543

0.341060221143393

$-1.9962115811219$

$-0.43141201807045$

0.245943298655885

$-1.59915760645132$

$-3.04329757211672$

$-2.19957311869648$

4.35283861210127

0.0894090693497365

$-5.61070977717332$

$-6.99623069053809$

$-7.95679631051658$

$-5.02547265146853$

$-6.43701672236178$

$-0.60907461810606$

$-0.134719858849978$

2.03379781945418

$-4.6010003814101$

$-4.46568779300636$

$-2.58425414279581$

6.8579089793615

4.45153645701015

6.17579851792792

7.83453629762552

5.15760084382157

5.84661147848919

8.50032728853189

7.5078735148003

9.09576238982968

11.2439260915642

9.23516005509561

12.3888806144132

8.06662274064481

11.3873405806894
1.24973401349362

0.331180627521869

$-0.529144756664611$

$-0.779106643384194$

$-1.22887819238817$

$-0.668056802444964$

$-1.52326721450433$

$-1.06732512327113$

0.105066000198598

$-0.556847901232874$

$-0.524190186414996$

0.558725969357907

$-0.28482667362296$

$-0.0893824001489402$

0.574139838015778

0.641802237773514

$-0.170469063711747$

$-0.0020654568061702$

$-0.0809807108134983$

$-0.162642978325762$

$-0.264616445499008$

3.69899384899172

$-0.492686078735562$

$-1.02702078424509 \mathrm{v}$

$-1.47817927003464$

$-0.520984902053338$

$-1.19880047978166$

0.452128689314895

$-0.846684435164739$

$-0.790310860156317$

$-0.645652800025356$

$-0.789257322356466$

$-0.596611286832089$

$-0.730828723790309$

$-0.426771967800393$

0.529724511867496

1.4722160655574

$-1.44595611524889$

$-2.43773628890699$

0.475928800568791

$-1.48393755207966$

$-0.00568940079531423$

0.710700048603213

$-1.96260874802742$

$-0.0896069302212548$

$-1.53280837257707$

$-1.61227859769491$

Chmielewski, Pawlicki, Sprutta, Szterenberg, Latos-Grażyński 
$-7.9602837702248$

$-6.47307268832363$

$-9.54010357786684$

$-7.76702021184398$

$-9.04279648463682$

$-11.148484207567$

$-8.65721045926572$

$-12.0191689500995$

$-10.7835865277951$

4.72951863787373

5.34660886287152

5.93543538796563

7.51855261217777

6.3785562664714

8.52820503647723

7.95614332861141

4.12181949876898

$-3.02742063832439$

9.67022821495583

8.87919237092847

0.702690987637084

$-3.40696843784686$

7.59305680873233

10.8056452140277

9.62203959374476

12.83218758928

$-3.35274819049999$

$-5.57666413202206$

$-8.78126898738472$

$-9.74863503812216$

$-7.53067822234344$

$-6.96830575171751$

$-10.2753334648003$

$-13.2345777557557$

$-12.8516226155087$

4.37938152676549

6.50768350659278

9.49966317444733

7.37238745382591

$-7.63347398320106$

13.4428042188202

9.08130519332039
9.24161437684168

$-4.67961720587642$

3.69798732666169

$-6.54735758246464$

$-8.32942718050015$

$-8.69480437559094$

$-5.07962939814493$

$-7.26380916324469$

$-5.46924527834376$

$-6.69773461326294$

$-8.80836334571243$

$-10.8356075580235$

$-12.1818601873906$

$-8.11618411525966$

$-11.5076690997119$

$-9.48483718385096$

8.30423982960214

0.635578854696415

0.259864414168823

3.83749908241049

$-9.24697010144226$

$-8.55045841134051$

7.16308032750008

9.45755587188716

4.21868092664363

6.51319809615069

10.084233226531

13.3416850898791

13.5086443754912

10.3728072948686

7.11596644109392

12.3888806144132

$-10.0762335693999$

$-9.04110712921239$

$-5.83609398406099$

$-9.49305754818795$

$-12.818596898067$

$-10.8155935502982$

$-7.49202011462511$

4.14160045288502

9.14445281962788

$-13.4963777391491$
$-2.3215534211955$

$-0.779840184956608$

$-3.18386352725571$

$-0.0395725217857082$

0.689283571467627

$-0.154294959740472$

$-1.63150153257112$

$-1.73664980366155$

$-2.46386668606615$

$-0.400789766428382$

0.549704511471013

1.48628869082625

0.488922983870115

$-1.41940868691555$

$-1.46503065261132$

$-2.41280187660381$

$-1.73607614186232$

$-2.06757615401169$

0.0630546181956967

0.0724641677750165

$-1.67798747614194$

$-1.91384212884357$

2.45517616384268

2.41537870476406

3.3780978739019

$-3.4248968365928$

1.52825511839394

1.43527382521109

$-0.892203632242659$

3.11547233527018

$-3.02371170236847$

$-0.0896069302212548$

1.42621087387925

$-0.998981125966392$

$-3.29824545486942$

2.47195137412643

2.42335778825885

$-3.40157413301193$

$-3.34750554450226$

1.67133370318158

$-0.526635061776639$

$-0.513548474832372$ 
Table S4. Ring points for 8 (xyz).
$-6.02286004988579$
$-1.75760001375253$
1.42170518893275
3.08638954778429
6.40069783964265
$-2.5866157221855$
2.17314315144
$-0.923711070843674$
10.2056350303516
$-6.53515232074863$
$-9.889383941151$
6.93565958822916

1.13160095045624

2.78936868489684

6.04069307843387

1.90214638363114

$-1.23522041617656$

$-1.88018552931159$

$-2.91978375630884$

$-6.11722559087584$

6.82983226363696

10.2209811981948

$-6.88623487917829$

$-10.1445480567925$
0.164510088131607

$-0.464667562852007$

$-0.987821105920786$

$-0.219848425479791$

$-0.102276900206524$

$-0.3689632265304$

$-0.212350364203034$

$-0.95467166588835$

$-0.482935928469614$

$-0.806132832180849$

$-0.888436179340119$

$-0.463335455823462$ 


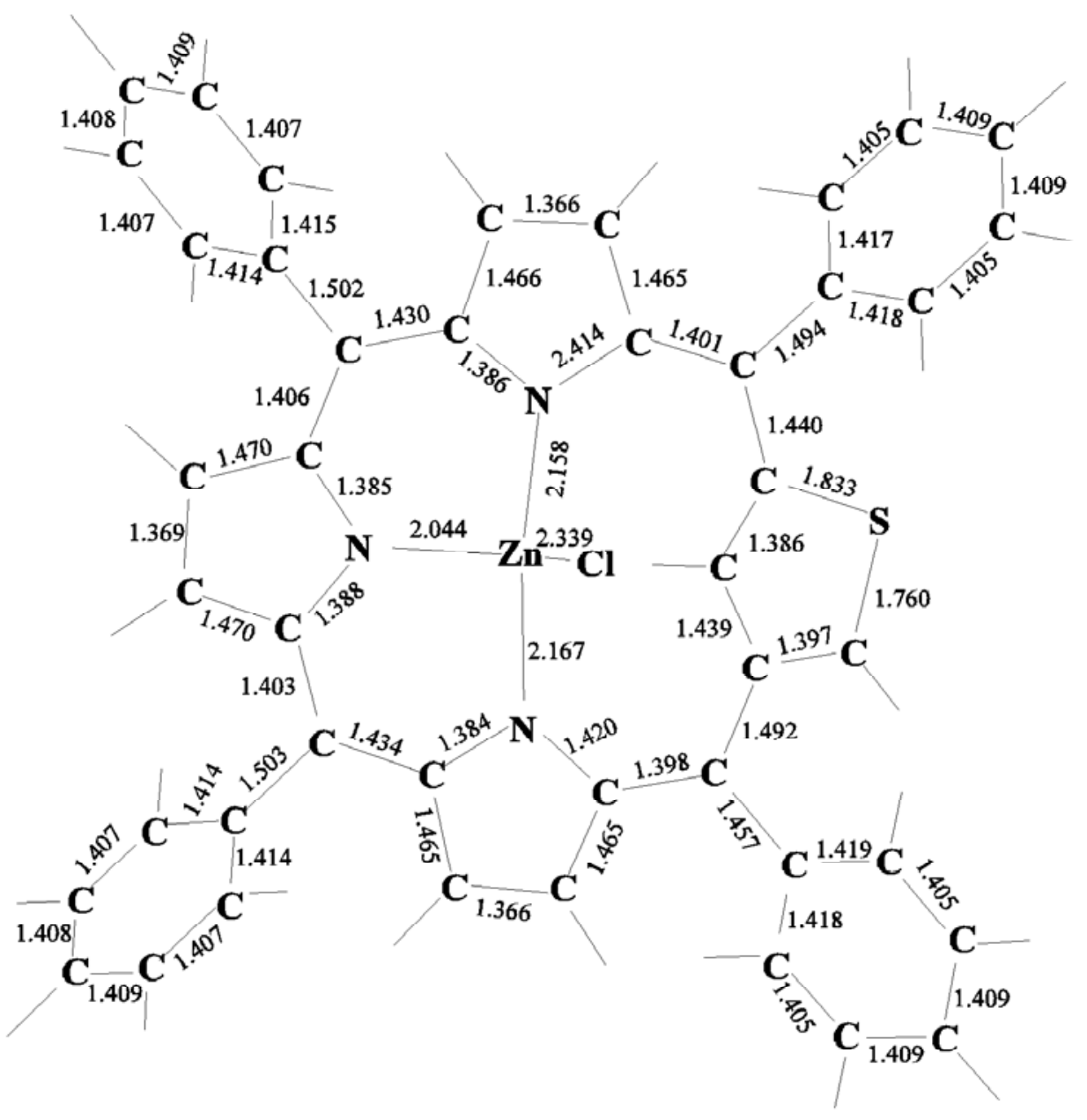

Figure S2. Bond lengths for DFT optimized geometry of 9 
Table S5. Cartesian coordinates for DFT optimized geometry of $\mathbf{9}$.

\begin{tabular}{|c|c|c|c|}
\hline & & & \\
\hline C & 7275 & -2.680692 & \\
\hline & & -2.404769 & \\
\hline & & & \\
\hline & 317 & .029576 & \\
\hline & & & \\
\hline & 66 & & \\
\hline & 067 & & \\
\hline & 11 & & \\
\hline & 65 & & \\
\hline & 52 & & \\
\hline & 082 & & \\
\hline & 38 & & \\
\hline & 8 & & \\
\hline & 2 & & \\
\hline & 1.2 & & \\
\hline & 2.4 & & \\
\hline & 2.2 & & \\
\hline & 3.2 & & \\
\hline & & & \\
\hline & & & \\
\hline & & & \\
\hline & & & \\
\hline V & & & \\
\hline$Z n$ & -0.2 & & \\
\hline & & & \\
\hline C & & & \\
\hline & 0 & -2.2 & \\
\hline & 7 & & \\
\hline & & -1. & \\
\hline & & & \\
\hline & -7.5 & -2.3 & \\
\hline & 9 & -4.8 & \\
\hline C & & & \\
\hline & 1.0 & -7.1 & \\
\hline & & -7.5 & \\
\hline & 2.7 & -6.6 & \\
\hline 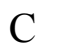 & & & \\
\hline $\mathrm{C}$ & 4.6 & & \\
\hline $\mathrm{C}$ & & & \\
\hline $\mathrm{C}$ & 6.4 & 3.0 & \\
\hline $\mathrm{C}$ & & 2.4 & \\
\hline 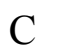 & & & \\
\hline $\mathrm{C}$ & & & \\
\hline $\mathrm{C}$ & -1.5 & & -0 . \\
\hline $\mathrm{C}$ & -1.1 & 5.678500 & \\
\hline 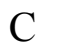 & & & \\
\hline 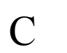 & -2.292481 & 7.566350 & -0.12445 \\
\hline
\end{tabular}

Chmielewski, Pawlicki, Sprutta, Szterenberg, Latos-Grażyński 


$\begin{array}{lrrr}\mathrm{C} & -2.722308 & 6.727912 & -1.171275 \\ \mathrm{C} & -2.355419 & 5.369247 & -1.186155 \\ \mathrm{H} & -1.372720 & -5.006627 & -1.074587 \\ \mathrm{H} & -3.705327 & -3.689470 & -0.896274 \\ \mathrm{H} & -5.313710 & 1.290436 & 0.173131 \\ \mathrm{H} & -4.054601 & 3.666846 & 0.156219 \\ \mathrm{H} & 0.992951 & 5.130978 & -0.868611 \\ \mathrm{H} & 3.387430 & 3.921335 & -1.012150 \\ \mathrm{H} & 1.243469 & -0.691611 & -1.374012 \\ \mathrm{H} & -4.627778 & -2.528398 & 1.724645 \\ \mathrm{H} & -6.978794 & -3.343836 & 1.787882 \\ \mathrm{H} & -5.435208 & -0.466526 & -1.978749 \\ \mathrm{H} & -7.788480 & -1.275307 & -1.921410 \\ \mathrm{H} & -0.189742 & -5.439882 & 1.138320 \\ \mathrm{H} & 0.508341 & -7.823465 & 1.154913 \\ \mathrm{H} & 2.406751 & -8.588133 & -0.282915 \\ \mathrm{H} & 3.601550 & -6.936123 & -1.731925 \\ \mathrm{H} & 2.898315 & -4.554293 & -1.749679 \\ \mathrm{H} & 4.412837 & 3.079245 & 1.222139 \\ \mathrm{H} & 6.786386 & 3.815215 & 1.239891 \\ \mathrm{H} & 8.451508 & 2.724295 & -0.273793 \\ \mathrm{H} & 7.715116 & 0.886812 & -1.801749 \\ \mathrm{H} & 5.338952 & 0.160686 & -1.824951 \\ \mathrm{H} & -0.508938 & 5.270368 & 1.686425 \\ \mathrm{H} & -1.157724 & 7.672484 & 1.720650 \\ \mathrm{H} & -3.336966 & 7.129118 & -1.973804 \\ \mathrm{H} & -2.679694 & 4.729174 & -2.003203 \\ \mathrm{H} & 3.288326 & -3.603563 & 1.153390 \\ \mathrm{H} & -8.572992 & -2.720071 & -0.036372 \\ \mathrm{H} & -2.578257 & 8.615478 & -0.110288\end{array}$


Table S6. Parameters of Selected Bond Critical Points (BCPs) Obtained from the AIM Analyses of $\mathbf{9}$ (au or dimensionless) ${ }^{\mathrm{a}}$

\begin{tabular}{lcccc}
\hline & $\rho$ & $\nabla_{\rho}^{2}$ & $\mathrm{H}$ & $\mathrm{B}$ \\
\cline { 2 - 5 } $\mathrm{Zn}-\mathrm{Cl}$ & 0.0471 & 0.3002 & 0.0059 & 0.2245 \\
$\mathrm{Zn} \cdots \mathrm{C}(21)$ & 0.0148 & 0.0569 & 0.0040 & 0.0101 \\
$\mathrm{Zn}-\mathrm{N}(24)$ & 0.0525 & 0.3663 & 0.0062 & 0.0725 \\
$\mathrm{Zn}-\mathrm{N}(23)$ & 0.0692 & 0.4856 & -0.0013 & 0.0741 \\
$\mathrm{Zn}-\mathrm{N}(22)$ & 0.0512 & 0.3572 & 0.0067 & 0.0711 \\
$\mathrm{C}(4)-\mathrm{C}(5)$ & 0.2917 & -0.7793 & -0.2925 & 1.5443 \\
\hline
\end{tabular}

${ }^{a}$ Explanation of symbols: $\rho$, electron density at BCP; $\nabla_{\rho}^{2}$, Laplacian; $\mathrm{H}$, total energy density; B, Wiberg bond index. 